\title{
HIGH STRENGTH, LOW VOLTAGE MICROFABRICATED ELECTROADHESIVES ON NON-CONDUCTIVE SURFACES
}

\author{
Abraham Simpson Chen, Alexi Charalambides, and Sarah Bergbreiter* \\ Department of Mechanical Engineering, University of Maryland, College Park, USA \\ The Institute for Systems Research, University of Maryland, College Park, USA
}

\begin{abstract}
This work demonstrated microfabricated all-polymer electroadhesive devices, which obtained a maximum shear strength of $78 \mathrm{kPa}$ at $200 \mathrm{~V}$ on a glass slide; these shear strengths are higher than previous work when operated at voltages an order of magnitude lower. A mold was microfabricated in silicon with features across multiple length scales, from centimeters to microns. The mold was refilled using conductive and dielectric polymers to decrease device stiffness over traditional metal electrodes, promoting a more conformal contact between the adhesive layer and substrate. Parylene was deposited to create a thin compliant dielectric between the electrodes and substrate, thereby reducing the voltage required to turn on the adhesives. Results show the effect of compliance on adhesive shear strength as well as the effect of gap spacing and number of electrodes.
\end{abstract}

\section{INTRODUCTION}

Electroadhesives are simple in nature; conductive electrodes coated with a dielectric material induce charge on a surface and adhere using electrostatic forces. Electroadhesion has been widely used for handling flat silicon wafers by means of rigid metal electrodes [1]. Recently, there have been developments to integrate electroadhesion into wall climbing robots [2], [3], where the adhesion can be easily controlled via voltage to turn on or off adhesion. Unlike wafers, these robots need to be able to adhere to relatively rough surfaces.

Previously, electroadhesives for wall climbing purposes have been made with metal deposited on Mylar films [2] or metal electrodes embedded in polymer [2], [3]. However, these methods produce dielectric layers that were typically tens to hundreds of microns thick which hindered shear strength at low voltages [4]. Other works have studied the effect of electrode geometries [3], [5], dielectric thickness [1], and adhesion substrate [2], [3], [6], but have been limited in shear strength, have required kilovolts to operate, or had electrode gaps on the order of millimeters to centimeters (thereby limiting the minimum adhesive sizes). The best shear strength obtained on a nonconductive surface in prior work was $62 \mathrm{kPa}$ at $5 \mathrm{kV}$ on glass [6]. Prior to this, others have only achieved a maximum $4.2 \mathrm{kPa}$ at $4 \mathrm{kV} \mathrm{[2].}$

Other approaches to adhesion have used inspiration from biology to create gecko-like adhesives with maximum shear strengths up to $300 \mathrm{kPa}$ on glass surfaces [7], [8], [9]. Geckos have hierarchical setae hairs on their toes that stick to surfaces by van der Waal forces. This, however, requires complex loading and peeling mechanisms to obtain strong adhesion, while being capable of detaching with minimal force. The Stickybot, which imitates a gecko's adhesive microstructures, employs directional polymerstalks that adhere primarily by van der Waals forces. When loaded onto a surface, the stalks' directional adhesion allows it to stick when pulled tangentially in one direction but releases when pulled in the opposite direction [10]. However, the adhesives often require cleaning and can not be turned on and off for more complex maneuvers.

Microfabrication provides the ability to create thin dielectric layers that reduce operating voltage while maintaining the ability to turn on and off adhesion. Gecko adhesives and the best

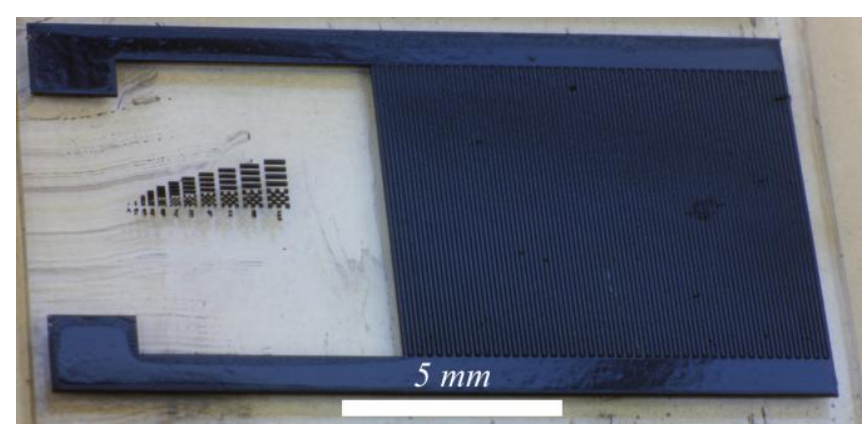

Fig. 1: Fabricated electroadhesive device.

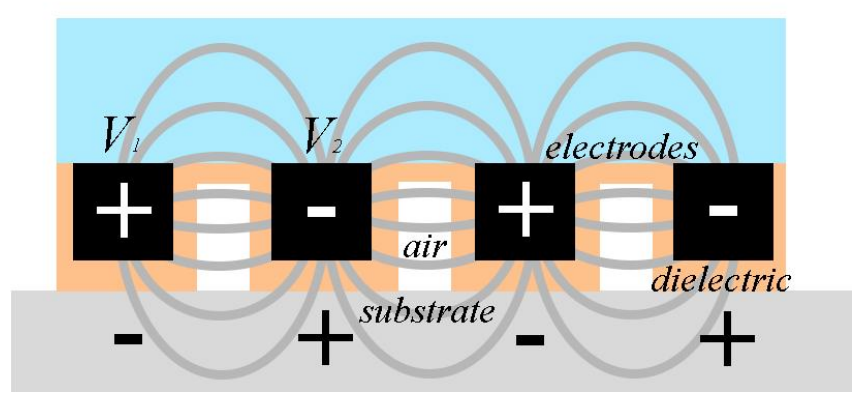

Fig. 2: Operation principle of electroadhesive device.

electroadhesives have demonstrated that compliance is key to the ability to conform and better adhere to rough substrates [6, 7]. Therefore, this work uses patterned conductive elastomer electrodes with a parylene dielectric to both improve adhesion and reduce operating voltage. An electroadhesive device created by this process can be seen in Fig. 1.

\section{ELECTROADHESIVE DESIGN}

Electrostatic adhesion utilizes the attraction between two oppositely charged surfaces separated by a dielectric layer. When adhering to nonconductive surfaces, two interdigitated electrodes are used to polarize the substrate, Fig. 2. In this case, Koh et al. developed a simple model based on the electrostatic attraction between the electrodes and surface as well as the fringing fields generated between the electrodes, Eq. 1 [4].

$$
F_{e}=\frac{\epsilon_{o} \epsilon_{r} w L V^{2}}{2 d^{2}}+\frac{0.265 \epsilon_{o} \epsilon_{r} w^{0.5} L V^{2}}{g^{1.5}}
$$

$V$ is the applied voltage, $w$ and $L$ are the width and length of the electrodes, $d$ is the distance between the electrodes and the substrate, $g$ is the gap between the pair of electrodes, $\epsilon_{0}$ is the permittivity of a vacuum, and $\epsilon_{r}$ is the dielectric constant (assuming that the same dielectric exists between the electrodes and the substrate).

Electric field clearly has a large effect on this electrostatic adhesive force. Previous work generally used dielectric thicknesses 
on the order of tens of microns [2-6]. Reducing dielectric thicknesses to the order of microns can proportionally reduce operating voltage requirements. In addition, this model shows that reducing the gap between electrodes can also improve adhesion. Microfabrication can be used to address both of these design goals.

However, electrostatic force is not the only factor that contributes to adhesion. In practice, the distance between electrodes and substrate varies across the interface due to non-flat surfaces. Results from work in gecko adhesives show that rigid materials exacerbate this effect [11], so conductive elastomer electrodes were used to reduce device stiffness in order to promote conformal contact.

In this work, three types of devices were fabricated to investigate electroadhesive shear strength. Non-patterned devices are simply conductive elastomers coated with a parylene dielectric. These devices were used to explore the effect of dielectric thickness and compliance on adhesion. "Type A" devices used patterned electrodes to adhere to non-conductive substrates and varied the gap between electrodes. Each Type A device had only 4 interdigitated electrode pairs. "Type B" devices were also patterned and varied the number of interdigitated electrodes. The gap was maintained at $10 \mu \mathrm{m}$. The device surface area was held constant at $1 \mathrm{~cm}^{2}$ for all three device types.

\section{FABRICATION}

Conductive electrodes in all three devices were made from a conductive polymer prepared using carbon black nanopowder (Alfa Aesar), Sylgard 184 polydimethylsiloxane (PDMS), and hexane. The carbon was mixed with PDMS at a ratio of $10 \mathrm{wt} \%$ carbon to $90 \mathrm{wt} \%$ PDMS, which was qualitatively found to be both conductive and elastic. The PDMS itself was prepared using a base to curing agent ratio of 10:1. Hexane was added to the mixture at a weight ratio of 2.5:1 hexane to PDMS to decrease viscosity, which helped facilitate mixing and mold refilling for the patterned adhesives. This mixture will be referred to as C/PDMS in the following sections.

\section{Non-patterned adhesives}

The non-patterned adhesives were prepared by pouring the C/PDMS mixture over a glass plate and applying a blade to set the thickness. The mixtures were cured in a vacuum oven at $90 \mathrm{C}$ for 20 minutes. Samples were then cut to size with a razor blade and coated with parylene C using the Parylene Deposition System Model 2010 from SCS. A small section of the device was covered with tape to later interface with the voltage source.

\section{Type $A$ and $B$ patterned adhesives}

The fabrication process for the patterned devices, Fig. 3, was based on previous work for tactile sensors [12]. First, $250 \mathrm{~nm}$ of silicon dioxide was deposited on a bare silicon wafer using PECVD, patterned, and used as a mask for a deep reactive ion etch (DRIE). Trenches were etched to a depth of approximately $30 \mu \mathrm{m}$. A fluoropolymer solution (DuPont) was used as an anti-stick layer for subsequent polymer processing. It was poured over the mold and vacuumed for 5 min to ensure a conformal coating. Then, it was spun for $60 \mathrm{~s}$ at $1000 \mathrm{rpm}$, and cured for $5 \mathrm{~min}$ at $145 \mathrm{C}$.

The C/PDMS was then poured over the mold and vacuumed for 3 minutes to remove air bubbles, ensure a conformal refill, and evaporate the hexane. Molded structures fabricated on a separate wafer with $100 \mu \mathrm{m}$ trenches show features down to $10 \mu \mathrm{m}$ are refilled and features down to $20 \mu \mathrm{m}$ have high yields, Fig. 4. Next, a conventional razor blade was squeegeed over the wafer surface to planarize the C/PDMS, after which it was cured on a hot plate for 15 minutes at $120 \mathrm{C}$.

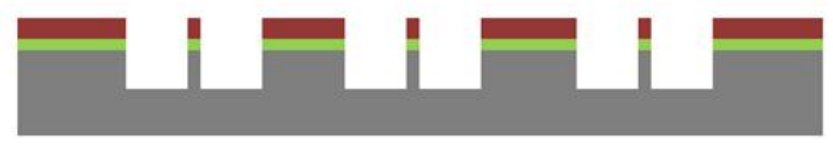

(a) Pattern mask and perform DRIE to create mold

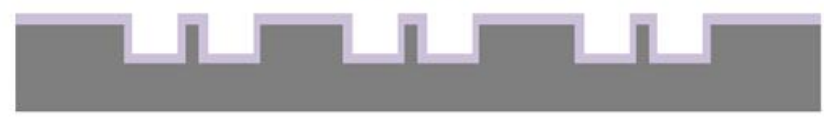

(b) Coat with Teflon

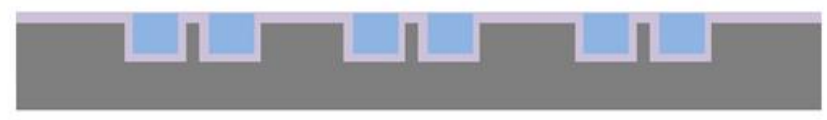

(c) C/PDMS refill and planarization

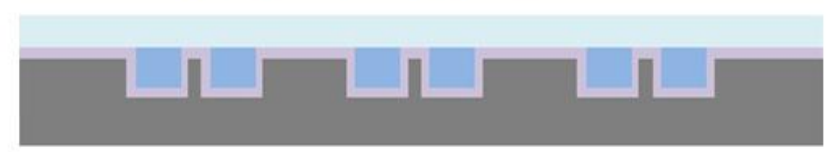

(d) PDMS spin coat

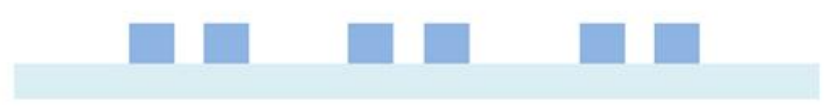

(e) Peel device

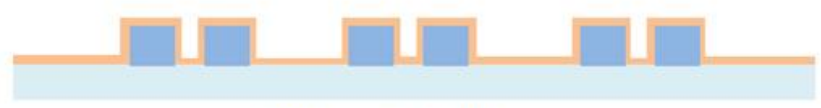

(f) Deposit parylene

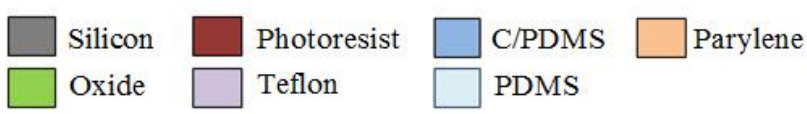

Fig. 3: Microfabrication flow chart.

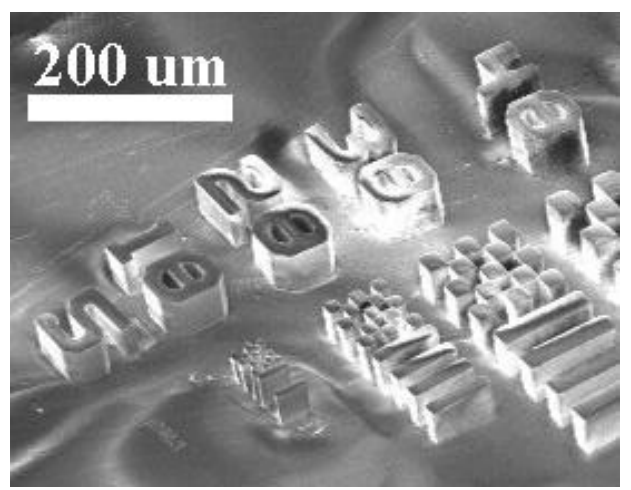

Fig. 4: Scanning electron microscope image of the refilled resolution marks. Aspect ratios of up to 10:1 were achieved.

Next, PDMS was poured over the mold and placed in vacuum for 15 minutes to remove air bubbles and ensure a strong bond between the PDMS and C/PDMS. It was spun for 30 seconds at $250 \mathrm{rpm}$ to attain a thickness of $400-500 \mu \mathrm{m}$, after which it was placed on a hot plate for $15 \mathrm{~min}$ at $120 \mathrm{C}$. Note that the PDMS was prepared with 20:1 base to curing agent ratio in order to minimize out-of-plane warping; warping was observed for PDMS ratios of 10:1, and may have been due to residual thermal stresses during 
curing or non-uniform dispersion of polymer chains during spinning [13].

Once cured, the PDMS layer was then gently peeled away, bringing with it the adhered conductive polymeric features. This sheet was manually cut into individual die, and finally coated with parylene $\mathrm{C}$ to act as a thin film dielectric.

\section{TEST SETUP}

Fig. 5 shows the test setup used to measure shear strengths for the fabricated devices. Samples were placed on the substrate and preloaded with a $20 \mathrm{~g}$ mass placed on top of the device (i.e. a $2 \mathrm{kPa}$ pre-load force for a $1 \mathrm{~cm}^{2}$ sample). The substrate was a silicon wafer for the non-patterned adhesives and a glass slide for the patterned adhesives. A voltage was applied between the substrate and the adhesive for the non-patterned samples and between the two electrodes in the patterned adhesives. This voltage was varied for the non-patterned adhesives and fixed at $200 \mathrm{~V}$ for both the Type A and B patterned adhesives. This voltage was applied for 2 min to ensure that the surface was fully polarized. The pre-load mass was then removed and the shear force on the sample was slowly increased via a pulley and applied mass. Shear force was increased until failure.

\section{RESULTS \& DISCUSSION}

In Fig. 6, various C/PDMS thicknesses were used to study the effect of adhesive compliance on adhesion strength. These adhesives were tested at $75 \mathrm{~V}$ with a parylene dielectric thickness of $1.1 \mu \mathrm{m}$. Each point in Fig. 6 represents the maximum shear strength a single device was able to achieve over 3 trials. Even though these devices were adhered to the smooth surface of a polished silicon wafer, adhesive thickness (and therefore compliance) has a large effect on the shear strength at failure. While absolute shear strengths are large for these devices, it is important to note that these non-patterned adhesives can only adhere to conductive substrates.

Non-patterned adhesives were also used to investigate the effect of varying the parylene dielectric thickness on adhesion strength, Fig. 7. As expected from Eq. 1, shear strength showed a quadratic relationship with voltage. In addition, shear strength decreased for larger parylene thicknesses; doubling dielectric thickness resulted in approximately doubling the voltage required to achieve the same shear pressure. At least three samples were tested three times at voltages that yielded shear strengths lower than $50 \mathrm{kPa}$ and at least three devices were tested one time for voltages that yield shear strengths above $50 \mathrm{kPa}$. These measurements were taken from devices with an average C/PDMS thickness of $470 \mu \mathrm{m}$.

For Type A patterned samples, three parametric devices were tested with different electrode gaps $(250 \mu \mathrm{m}, 100 \mu \mathrm{m}$, and $50 \mu \mathrm{m})$, while the electrode geometry and number of electrodes were held constant, Fig. 8; average shear strengths were $78 \mathrm{kPa}, 57 \mathrm{kPa}$, and $48 \mathrm{kPa}$, respectively. Maximum shear strength of $78 \mathrm{kPa}$ was obtained with the smallest fabricated electrode gap of $50 \mu \mathrm{m}$. The trend seen in Fig. 8 is expected, in part, due to the increased contribution of fringing fields to electrostatic force as the electrode gap decreases [4]. Decreasing the electrode gap from $250 \mu \mathrm{m}$ to 50 $\mu \mathrm{m}$ showed a $40 \%$ improvement in shear strength.

For Type B samples, the number of electrode pairs was varied $(83,45,20,10$, and 5) while an electrode gap of $10 \mu \mathrm{m}$ was held constant, Fig. 9. Each point represents the maximum shear strength a single device was able to achieve over 3 trials. It was found that as the number of pairs increased the shear strength decreased. This is because the rate at which shear strength increases as interdigitated electrode pairs increases (fringing field term) is less

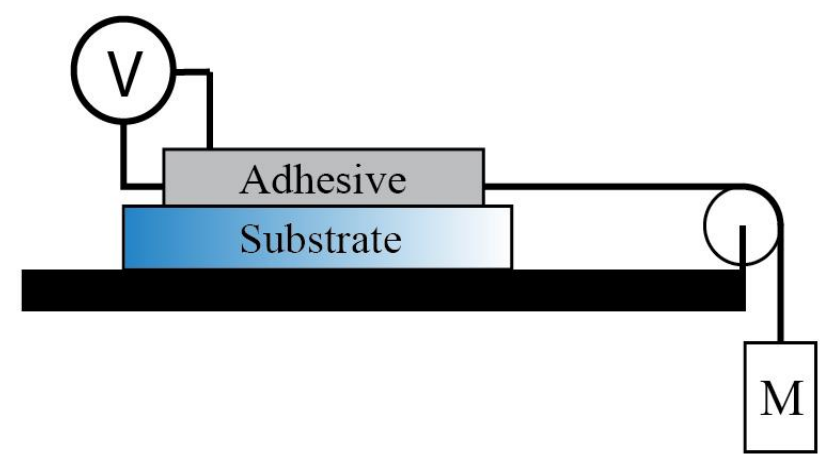

Fig. 5: Electroadhesive test setup.

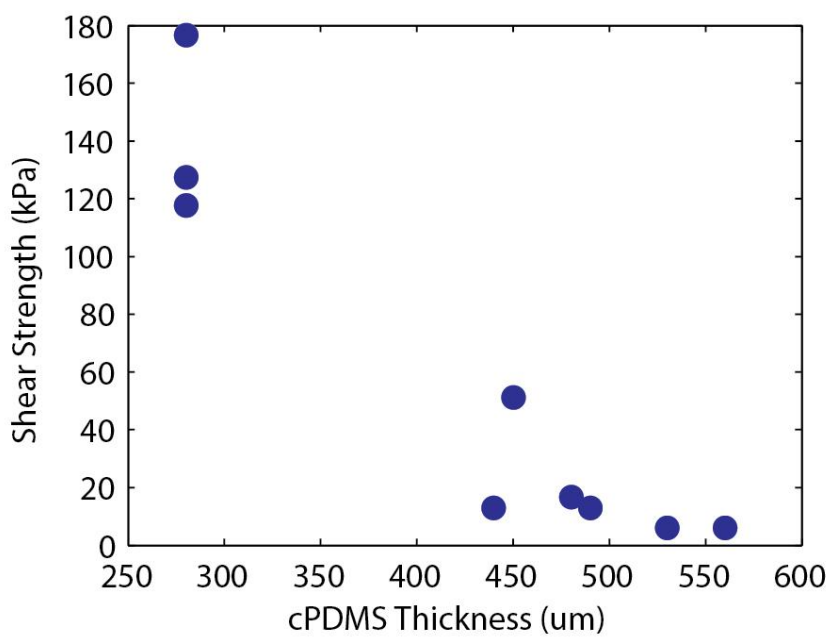

Fig. 6: Shear strength as a function of voltage, for various carbonPDMS thicknesses.

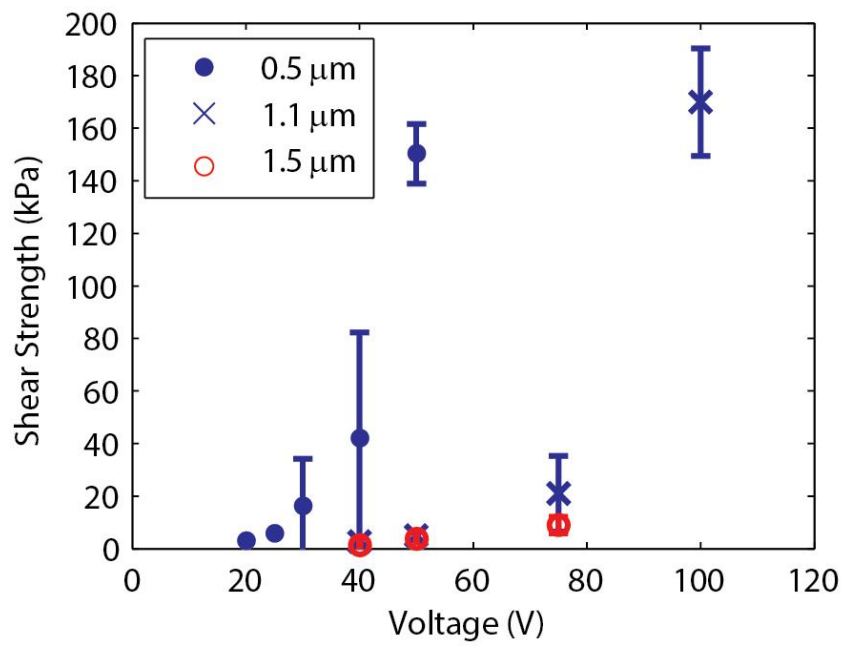

Fig. 7: Shear strength as a function of voltage for non-patterned conductive polymer, for various dielectric thicknesses.

than the rate at which shear strength decreases as electrode width decreases (direct field term).

By using parylene $\mathrm{C}$ as the dielectric layer and polymers as the bulk material, the dielectric thickness and device conformity were favorable for generating high shear strength at low voltages. This is a great improvement compared to most works that require kilovolts while only obtaining shear strength in the single $\mathrm{kPa}$. 


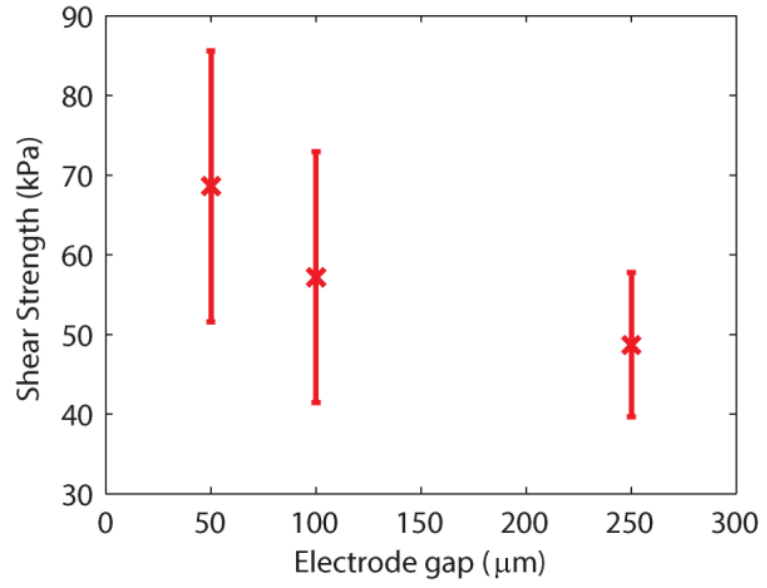

Fig. 8: Shear strength versus electrode gap $(250 \mu \mathrm{m}, 100 \mu \mathrm{m}$, and $50 \mu \mathrm{m}$ ) of the Type A devices at $200 \mathrm{~V}$. Deviation bars are from 3 tested devices.

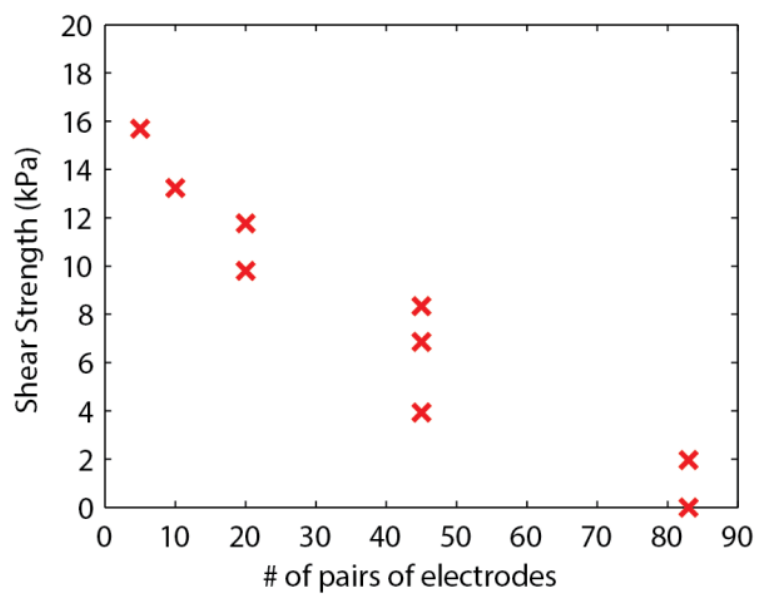

Fig. 9: Shear strength versus number of pairs of interdigitated electrodes of the Type B devices at $200 \mathrm{~V}$.

This fabrication process also supports electroadhesives that have over a hundred interdigitated fingers with electrode gaps as low as $10 \mu \mathrm{m}$. This is also because of the mold depth of $30 \mu \mathrm{m}$, which effectively reduces attraction between adjacent electrodes. Deeper molds will introduce a tradeoff between added electrode compliance and the possibility of electrode structures pulling in to adjacent electrodes. The dielectric breakdown of $1 \mu \mathrm{m}$ thick parylene (average for our devices) was measured to be at $300 \mathrm{~V}$, which is significantly higher than at $200 \mathrm{~V}$, the operating voltage of the Type A and B samples.

\section{CONCLUSION}

These results demonstrate the potential for microfabricated electroadhesive devices and for the first time, demonstrates electroadhesives that are comparable to the best gecko adhesives with shear strengths of approximately $300 \mathrm{kPa}$ [7]. The demonstrated design is advantageous due to its low voltage requirement, ability to adhere to nonconductive surfaces, and compliant to adhere to rough surfaces. In the future these adhesives can be used on small scale climbing robots and robotic grippers.

\section{ACKNOWLEDGEMENT}

This material is based upon work supported by NASA under Award \#NNX12AM02G. The authors would also like to thank the staff of the Maryland Nanocenter for their support.

\section{REFERENCES}

[1] K. Asano, F. Hatakeyama, and K. Yatsuzuka, "Fundamental study of an electrostatic chuck for silicon wafer handling," IEEE Transactions on Industry Applications, vol. 38, no. 3, pp. 840-845, 2002.

[2] H. Prahlad, R. Pelrine, S. Stanford, J. Marlow, and R. Kornbluh, "Electroadhesive robotswall climbing robots enabled by a novel, robust, and electrically controllable adhesion technology," in IEEE International Conference on Robotics and Automation, 2008. ICRA 2008. IEEE, 2008, pp. 3028-3033.

[3] J. P. D. Tellez, J. Krahn, and C. Menon, "Characterization of electroadhesives for robotic applications," in 2011 IEEE International Conference on Robotics and Biomimetics (ROBIO). IEEE, 2011, pp. 1867-1872.

[4] K. H. Koh, R. Kuppan Chetty, and S. Ponnambalam, "Modeling and simulation of electrostatic adhesion for wall climbing robot," in 2011 IEEE International Conference on Robotics and Biomimetics (ROBIO). IEEE, 2011, pp. 20312036.

[5] D. Ruffatto, J. Shah, and M. Spenko, "Optimization and experimental validation of electrostatic adhesive geometry," in Aerospace Conference, 2013 IEEE. IEEE, 2013, pp. 1-8.

[6] D. Ruffatto III, J. Shah, and M. Spenko, "Increasing the adhesion force of electrostatic adhesives using optimized electrode geometry and a novel manufacturing process," Journal of Electrostatics, 2014.

[7] A. G. Gillies and R. S. Fearing, "Shear adhesion strength of thermoplastic gecko-inspired synthetic adhesive exceeds material limits," Langmuir, vol. 27 , no. 18, pp. 11 278-11 $281,2011$.

[8] J. Krahn and C. Menon, "Electro-dry-adhesion," Langmuir, vol. 28, no. 12, pp. 5438-5443, Mar. 2012.

[9] O. Unver, A. Uneri, A. Aydemir, and M. Sitti, "Geckobot: a gecko inspired climbing robot using elastomer adhesives," in Robotics and Automation, 2006. ICRA 2006. Proceedings 2006 IEEE International Conference on, 2006, pp. 23292335.

[10] D. Santos, B. Heyneman, S. Kim, N. Esparza, and M. R. Cutkosky, "Gecko-inspired climbing behaviors on vertical and overhanging surfaces," in Robotics and Automation, 2008. ICRA 2008. IEEE International Conference on, 2008, pp. 1125-1131.

[11] Geim, A. K., et al. "Microfabricated adhesive mimicking gecko foot-hair." Nature materials 2.7 (2003): 461-463.

[12] A. Charalambides and S. Bergbreiter, "All-elastomer in-plane mems capacitive tactile sensor for normal force detection," in Sensors, 2013 IEEE. IEEE, 2013, pp. 1-4.

[13] P. Damman, S. Gabriele, S. Copp'ee, S. Desprez, D. Villers, T. Vilmin, E. Rapha“el, M. Hamieh, S. Al Akhrass, and G. Reiter, "Relaxation of residual stress and reentanglement of polymers in spin-coated films," Physical review letters, vol. 99, no. 3, p. 036101, 2007.

\section{CONTACT}

*sarahb@umd.edu, 301-405-6506 